\title{
Measuring the flavor asymmetry in the sea quarks of the proton
}

\author{
Paul E. Reimer*广 \\ Physics Division, Argonne National Laboratory, Argonne, Illinois 60439, USA \\ E-mail: reimereanl.gov
}

\begin{abstract}
The proton is a composite object made of fundamental, strongly-interacting quarks. Many of the features of the proton can be described by a simple picture based on three valence quarks bound by the exchange of gluons. However, protons are much more complex objects with the vast majority of their mass dynamically generated by Quantum Chromodynamics (QCD). This mass manifests itself through a "sea" of gluons and quark-antiquark pairs. By measuring DrellYan scattering, the Fermilab E-906/SeaQuest experiment will study the sea quark distribution in the proton and, in particular, the unusually large asymmetry between anti-up and anti-down quarks measured by earlier Drell-Yan experiments. This asymmetry cannot simply be generated through pair creation, but rather indicates an underlying, fundamental antiquark component in the proton. Using the same technique, E-906/SeaQuest will also investigate the differences between the antiquark distributions of the free proton and a proton bound in a nucleus. Nuclear binding is expected to modify the quark distributions and it has long been known that the overall quark distributions are different (the EMC effect). Surprisingly, present data suggests that the antiquark distributions and hence the sea distributions are not modified. To accomplish these goals, the experiment will used a $120 \mathrm{GeV}$ proton beam extracted from the Fermilab Main Injector. While the experiment will be taking advantage of equipment from earlier Drell-Yan experiments, the changes in kinematics of the experiment require several, significant upgrades to the spectrometer. The collaboration expects to begin data collection in fall 2010.
\end{abstract}

XVIII International Workshop on Deep-Inelastic Scattering and Related Subjects, DIS 2010 April 19-23, 2010

Firenze, Italy

\footnotetext{
*Speaker.

$\dagger$ Representing the Fermilab E-906/SeaQuest Collaboration: Abilene Christian, Academia Sinica, Argonne, Colorado, Fermilab, Ilinois, KEK, Kyoto, Los Alamos, Maryland, Michigan, National Kaohsiung Normal, New Mexico, RIKEN, Rutgers, Texas A \& M, JLab, Tokyo Tech and Yagamata.
} 
The quark composition of both the nucleon and the nucleus has been studied extensively with a variety of processes. In particular, electromagnetic deep inelastic scattering (DIS) has been used to elucidate a wealth of information on this structure, but lacks the basic ability to distinguish between quark and antiquark distributions within the nucleon. The Drell-Yan process provides a probe that is sensitive to the antiquark distributions of the interacting hadrons. The Drell-Yan process [1] to leading order in $\alpha_{s}$ is the annihilation of a quark in one hadron with an antiquark in a second hadron to form a virtual photon. The virtual photon decays into a lepton-antilepton pair which is detected. The cross section is dependent on the charge-squared-weighted sum of the quark and antiquark distributions in the interacting hadrons:

$$
\frac{d^{2} \sigma}{d x_{1} d x_{2}}=\frac{4 \pi \alpha^{2}}{9 s x_{1} x_{2}} \sum_{i \in\{u d c s\}} e_{i}^{2}\left[q_{1 i}\left(x_{1}\right) \bar{q}_{2 i}\left(x_{2}\right)+\bar{q}_{1 i}\left(x_{1}\right) q_{2 i}\left(x_{2}\right)\right],
$$

where $q_{1 i(2 i)}$ are the beam (target) quark distributions, the sum is over all quark flavors $(u, d, s$, $c$ ) and $e_{i}$ is the fractional quark charge. The fraction of the longitudinal momentum of the beam (target) carried by the participating quark is $x_{1(2)}$ and $s$ is the center-of-mass energy squared.

In a fixed, forward acceptance spectrometer, Drell-Yan scattering has a unique sensitivity to the antiquark distribution of the target hadron where the acceptance restricts the event kinematics to $x_{1} \gg x_{2}$. At very high values of $x_{1}$ where the antiquark distributions are suppressed by several orders of magnitude relative to the quark distributions. These beam valence quarks annihilate with antiquarks in the target, preferentially selecting the first term in (1). This feature has been exploited by several experiments to study antiquark distributions [2, 3, 4].

This paper will discuss measurements of the antiquark distributions in the proton and in nuclei. Building on this, it will motivate and describe a new experiment at Fermilab, E-906/SeaQuest [5]. E-906/SeaQuest is currently under construction and will begin collecting data in the fall of 2010 .

\section{Origins and Isospin Symmetry of the Light Quark Sea}

Initially, the quark sea was believed to originate through gluon splitting-starting with three valence quarks of the correct distribution, the remaining sea quarks could be dynamically generated through QCD evolution [6]. Fits to existing data, however, indicated the need intrinsic sea quark distributions as well [7]. Even though it was clear that the sea distributions were not purely dynamically generated, because of approximately equal mass of the $u$ and $d$ quarks, it was widely believed that the sea distributions were $\bar{d}-\bar{u}$ symmetric. The observation of a violation of the Gottfried Sum Rule [8] in New Muon Collaboration [9] proved this assumption to be incorrect.

The sensitivity of Drell-Yan scattering to antiquark distributions makes it an ideal probe of this asymmetry [10]. In leading order, assuming $x_{1} \gg x_{2}$ and the dominance of the $u \bar{u}$ annihilation term, the ratio (per nucleon) of the proton-proton to proton-deuterium Drell-Yan yields is

$$
\left.\frac{\sigma_{p d}}{2 \sigma_{p p}}\right|_{x_{1} \gg x_{2}}=\frac{1}{2}\left[1+\frac{\bar{d}\left(x_{2}\right)}{\bar{u}\left(x_{2}\right)}\right] .
$$

The next-to-leading order terms in the cross section provide a small correction to this ratio, as does the fact that the data may not all satisfy the $x_{1} \gg x_{2}$ condition. 

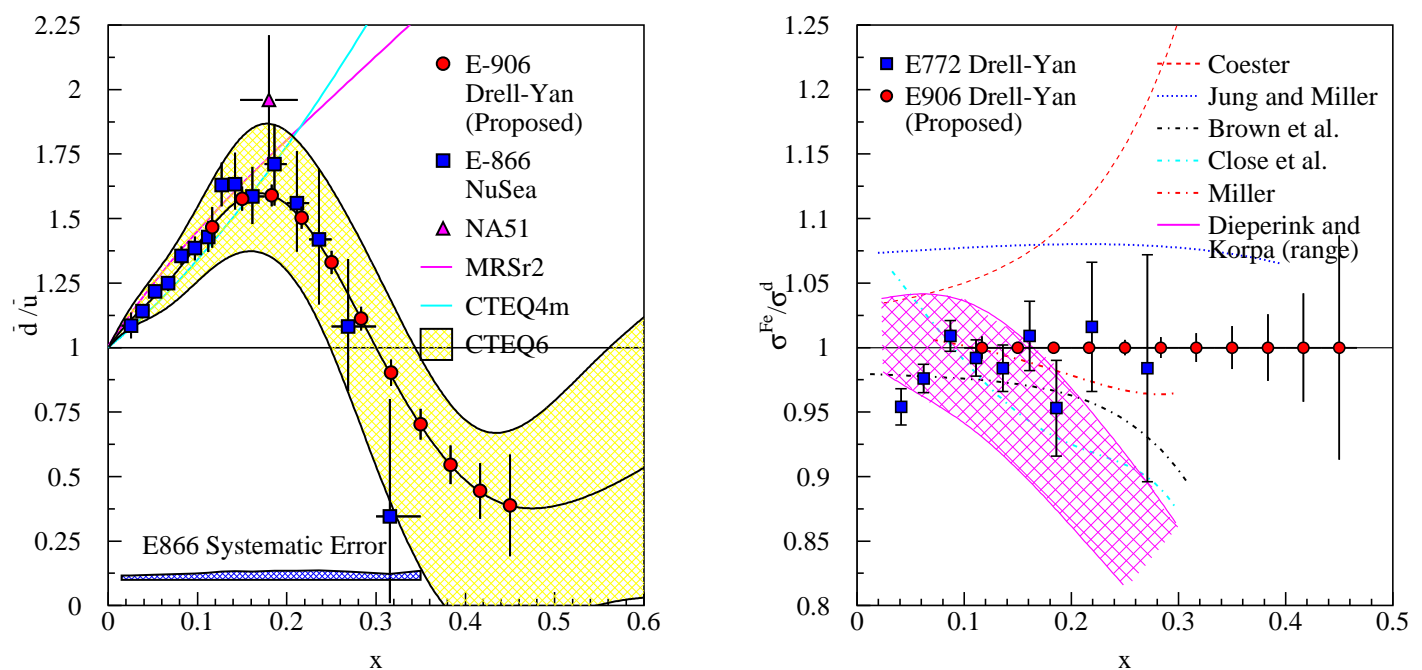

Figure 1: (Left) Measurement of $\bar{d}(x) / \bar{u}(x)$ by E-866/NuSea [2] (blue squares) and NA51 [12] (green triangles) are shown. The central curve shows the $\bar{d} / \bar{u}$ ratio and uncertainty from the CTEQ5M fit, which included the E-866/NuSea data. The red circles represent the expected statistical uncertainties of the E-906 data. [5]. (Right) Ratio of iron to deuterium Drell-Yan cross sections (blue squares) [4] and the expected sensitivity of E-906 (red circles). Curves based on several different models are also plotted [16, 17].

The Fermilab E-866/NuSea experiment used this sensitivity to measure $x$-dependence of the $\bar{d} / \bar{u}$ ratio with an $800 \mathrm{GeV} / \mathrm{c}$ proton beam incident on hydrogen and deuterium targets. From the measured ratio of Drell-Yan yields E-866/NuSea extracted the ratio $\bar{d}(x) / \bar{u}(x)$ shown in Fig. 1. While Eq. 1.1 is illustrative of the sensitivity, the actual E-866/NuSea extraction was done in nextto-leading order without the $x_{1} \gg x_{2}$ and $u \bar{u}$ dominance assumptions. The inclusion of the measured cross section ratios in global parton distribution fits [11] validated this extraction and completely changed the perception of the sea quark distributions in the nucleon. At moderate values of $x$ the data show more than $60 \%$ excess of $\bar{d}$ over $\bar{u}$, but as $x$ grows larger, this excess disappears. A purely perturbative sea would exhibit only a very small asymmetry between $\bar{d}$ and $\bar{u}$.

Non-perturbative explanations for the origin of the sea including meson cloud models, chiral perturbation theory or instantons can explain a large asymmetry $[3,13]$, but they fail to predict the return to a symmetric sea seen as $x \rightarrow 0.3$ and none predicts an excess of $\bar{u}$ over $\bar{d}$ as seen in the parton distribution fits [11]. As $x$ increases beyond 0.25 , the data become less precise. To help understand this region better, the Fermilab E-906 experiment has been approved to collect DrellYan data in this region. The expected statistical uncertainties of the E-906/SeaQuest experiment are shown in Fig. 1, based on a redesigned spectrometer. (See Sec. 3.)

\section{Antiquark Distributions of Nuclei}

The distributions of partons within a free and bound nucleon are different, an effect discovered by the European Muon Collaboration (EMC) in 1983 [14]. Almost all of the data on nuclear dependencies of quark distributions is from DIS experiments. Sea quark nuclear effects may be entirely different, but DIS experiment would not be very sensitive to this. 


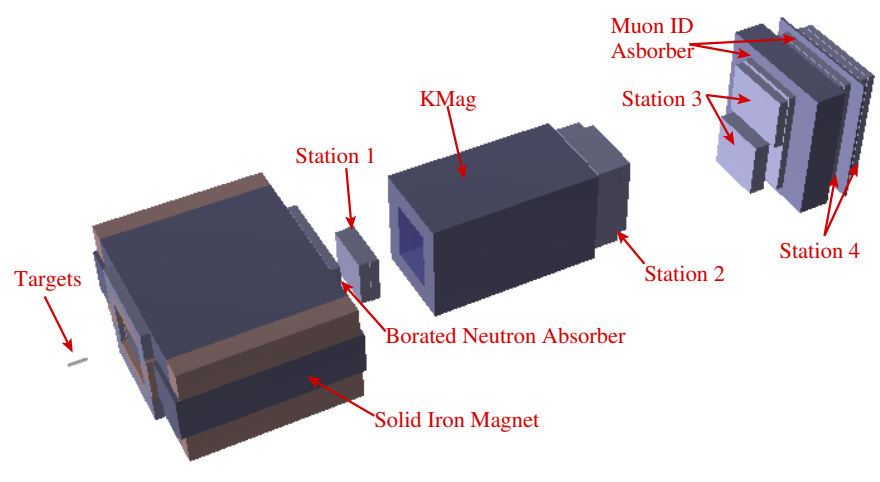

Figure 2: Schematic view of the E-906/SeaQuest detector. Each of the labeled stations consists of a tracking chamber and a set of hodoscopes for triggering.

In fact, no modification to the sea quark distributions was observed by Fermilab E-772 [4]. (See Fig. 1.) Several models of nuclear binding, which can explain the EMC effect, rely on the exchange of virtual mesons [15]. Based on these models a significant enhancement in the antiquark distributions in nuclei was expected [16]. The lack of sea-quark nuclear effects prompted a number of newer models which are also shown in Fig. 1 [17]. For $x>0.2$, the E-772 statistical uncertainties allow significant freedom for these models. E-906/SeaQuest will provide data on the nuclear dependence of the sea quarks with the statistical precision shown in Fig. 1.

\section{Fermilab E-906/SeaQuest}

The E-906/SeaQuest experiment will achieve the sensitivities shown in Fig. 1 using a $120 \mathrm{GeV}$ proton beam from the Fermilab Main Injector. This lower beam energy has two major advantages: (1) the cross section is proportional to the inverse of the center-of-mass energy squared, $s$. The lower energy beam will increase the cross section, and (2) the predominant correlated dimuon background is from the dimuon $J / \psi$ decay. $J / \psi$ production scales approximately with the centerof-mass energy, and thus background will be decreased. While reducing the energy results in a larger cross section, at some point, the energy will become so low that the interaction is no longer between uncorrelated partons. With a $120 \mathrm{GeV}$ beam, this experiment is still well above this limit. In addition, as the energy decreases, the background from uncorrelated pion decay will increase.

The Fermilab E-906/SeaQuest spectrometer [5] is modeled after its predecessors. To take advantage of the lower beam energy, the spectrometer must be shortened in order to maintain the same transverse momentum acceptance. For much of the spectrometer, this was done by moving the locations of spectrometer elements. The first, focusing magnet had to be shortened as well. This was acheived by using existing coils and switching to a closed-aperture, solid-iron design. A schematic view of the E-906/SeaQuest spectrometer is shown in Fig. 2. The spectrometer is being assembled in the NM4 (KTeV) hall at Fermilab. Production with low intensity beam will begin in Fall, 2010. The experiment will collect data for an equivilent of two years at full luminoisty. 


\section{Conclusions}

Drell-Yan experiments have contributed to the understanding of the antiquark distributions of the nucleon and their modifications by a nuclear environment. The Fermilab E-906/SeaQuest experiment will revisit many of these measurements with improved statistical precision and greater kinematic reach. The E-906/SeaQuest experiment is under construction and will begin collecting data in the fall of 2010. These data will allow E-906/SeaQuest to extend the measurement of $\bar{d} / \bar{u}$ and also determine the nuclear dependence of the sea, with statistical precision that will challenge the current models of nuclear binding.

This work is supported in part by the U.S. Department of Energy, Office of Nuclear Physics, under Contract No. DE-AC02-06CH11357.

\section{References}

[1] S.D. Drell and T.-M. Yan, Phys Rev. Lett 25:316 (1970); S.D. Drell and T.-M. Yan, Phys Rev. Lett 25:902 (1970); and Christenson et al. Phys. Rev. Lett 25:1523 (1970).

[2] E.A. Hawkeret al. (Fermilab E-866/NuSea Collaboration) Phys. Rev. Lett. 80:3715 (1998); and R.S. Towell et al. (Fermilab E-866/NuSea Collaboration) Phys. Rev. D 64:052002 (2001).

[3] J.-C. Peng et al. (Fermilab E-866/NuSea Collaboration) Phys. Rev. D 58:092004 (1998).

[4] D.M. Alde et al. (Fermilab E-772 Collaboration) Phys. Rev. Lett. 64:2479 (1990).

[5] P.E. Reimer and D.F. Geesaman et al. (Fermilab E-906/SeaQuest Collaboration).

[6] M. Gluck, E. Reya, and A. Vogt, Z. Phys. C 48:471 (1990).

[7] M. Gluck, E. Reya, and A. Vogt, Z. Phys. C 53:127 (1992).

[8] K. Gottfried, Phys. Rev. Lett 18:1174 (1967).

[9] P. Amaudruz et al (CERN NMC Collaboration) Phys. Rev. Lett 66:2712 (1991); and M. Arneodo et al (CERN NMC Collaboration) Phys. Rev. D 50:R1 (1994).

[10] S.D. Ellis and W.J. Stirling Phys. Lett. B 256:258 (1991).

[11] J. Pumplin et al. (CTEQ Collaboration) JHEP 2002:012 (2002); A.D. Martin et al. Eur. Phys. J. C 39:155 (2005); and M. Gluck, E. Reya, and A. Vogt, Eur. Phys. J. C 5:461 (1998).

[12] A. Baldit et al. Phys. Lett. B 332:244 (1994).

[13] M. Albert, E.M. Henley and G.A. Miller Phys. Lett. b 47:396 (2000); N.N. Nikolaev et al. Phys. Rev. D 60:014004 (1999); A. Szczurek, J. Speth and G.T. Garvey Nucl. Phys. A 570:765 (1994); P.V. Pobylista et al. Phys. Rev. D 59:034024 (1999); and A.E. Dorokhov and N.I. Kochelev Phys. Lett. B 304:167 (1993).

[14] J.J. Aubert et al. (CERN EMC Collaboration) Phys. Lett. B 123:275 (1983).

[15] J. Carlson and R. Schiavilla Rev. Mod. Phys. 70:743 (1998).

[16] E.L. Berger, F. Coester adn R.B. Wiringa, Phys. Rev. D 29:398 (1984); E.L. Berger and F. Coester, Phys. Rev. D 32:1071 (1985); and F. Coester, Private Communication (2001).

[17] H. Jung and G.A. Miller Phys. Rev. C 41:659 (1990); G.E. Brown et al. Nucl. Phys. A 593:295, (1995); A.E.L. Diepering and C.L. Korpa, Phys. Rev. C 55:2665, May 1997; J.R. Smith and G.A. Miller Phys. Rev. Lett. $91: 212301$ (2003). 